\title{
Performance and scientific objectives of the SELENE (KAGUYA) Multiband Imager
}

\author{
Makiko Ohtake $^{1}$, Junichi Haruyama ${ }^{1}$, Tsuneo Matsunaga ${ }^{2}$, Yasuhiro Yokota ${ }^{1}$, \\ Tomokatsu Morota ${ }^{1}$, Chikatoshi Honda ${ }^{1}$, and LISM team \\ ${ }^{1}$ Planetary Science Department, Japan Aerospace Exploration Agency (JAXA), 3-1-1 Yoshinodai, Sagamihara, Kanagawa 229-8510, Japan \\ ${ }^{2}$ The National Institute for Environmental Studies (NIES), 16-2, Onogawa, Tsukuba, Ibaraki 305-8506, Japan
}

(Received April 2, 2007; Revised August 20, 2007; Accepted October 31, 2007; Online published April 9, 2008)

\begin{abstract}
The Multiband Imager (MI) is one of the 14 instruments for the Japanese SELENE (KAGUYA) mission. Goal of the SELENE (KAGUYA) mission is to understand origin and evolution of the Moon by obtaining global element and mineral compositions, topological structure, gravity field of the whole Moon, and electromagnetic and particle environment of the Moon. MI is designed to be a high-resolution multiband imaging camera with a spatial resolution in visible bands of $20 \mathrm{~m}$ and a spatial resolution in near-infrared bands of $62 \mathrm{~m}$ from the $100 \mathrm{~km}$ SELENE (KAGUYA) orbit altitude. The MI flight model has been manufactured and integrated. MTF, viewing vector, over-all sensibility, sensor linearity and electrical noise level ( $\mathrm{S} / \mathrm{N}$ estimation test) were measured, and the results indicate that the MI will provide sufficient MTF and low-noise data, just as estimated in the MI design phase. Operation and data analyses plans have been established, and related tools and algorithms have been developed and checked. One of MI scientific objectives is to investigate small but scientifically very important areas such as crater central peaks and crater walls and to investigate magnesian anorthosites.
\end{abstract}

Key words: Moon, SELENE, KAGUYA, Multiband Imager.

\section{Introduction}

Japanese SELenological and ENgineering Explorer (SELENE; it is also named "KAGUYA" after a Japanese folklore) mission launched in September 2007 (Kato et al., in print). Goal of the SELENE (KAGUYA) mission is to understand origin and evolution of the Moon by obtaining global element and mineral compositions, topological structure, gravity field of the whole Moon, and electromagnetic and particle environment of the Moon. SELENE (KAGUYA) satellite arrived at the Moon about one month after its launch and will continue its observation during nominal one-year mission.

The Lunar Imager/SpectroMeter (LISM) instrument is being developed for the SELENE (KAGUYA) mission and will provide us much higher spatial resolution, wavelength resolution and signal to noise ratio than that of past lunar missions. LISM consists of the three subsystems, the Terrain Camera (TC), the Multiband Imager (MI), and the Spectral Profiler (SP). The subsystems share some components and electronics including the Data Processing Unit (DPU), Data Processing and Control Unit (SDPCU) and Power Control and Distribution Unit (PCDU). Figure 1 schematically illustrates the LISM radiometer units. MI and TC sensors share a unit structure.

TC is a push-broom imaging camera with a spatial resolution of $10 \mathrm{~m}$ from the $100 \mathrm{~km}$ SELENE (KAGUYA) orbit altitude. It has two slant telescopes for stereo imag-

Copyright (c) The Society of Geomagnetism and Earth, Planetary and Space Sciences (SGEPSS); The Seismological Society of Japan; The Volcanological Society of Japan; The Geodetic Society of Japan; The Japanese Society for Planetary Sciences; TERRAPUB ing. TC stereoscopic observation and also single-eyed observation are planned for detailed investigation of surface features of the Moon. TC will cover the whole Moon during nominal one-year operation of SELENE (KAGUYA). Global, detailed investigation of the topography and geographical features of the Moon will be derived through TC data (Haruyama et al., 2008, this issue).

MI is a high-resolution multiband imaging camera consisting of visible and near-infrared sensors which have 5 visible and 4 near-infrared bands. MI takes push-broom imaging data by using selected 5 lines for MI-VIS and 4 lines for MI-NIR which are selected before launch. The spatial resolution of visible bands is $20 \mathrm{~m}$, and that of near-infrared bands is $62 \mathrm{~m}$ from the $100 \mathrm{~km}$ SELENE (KAGUYA) orbital altitude. We are going to observe the global mineral distribution of the lunar surface in nine band images of MI.

SP is a visible to near-infrared line-profiling spectrometer. Its primary scientific goal is to map the rock type and the mineral abundance/composition on the lunar surface globally through reflectance spectroscopy in the 500-2600 nm region with high spectral resolution (300 bands) and S/N. SP has a Cassegrain-type foreoptics and three detectors. Reflected light is separated into three spectral ranges (VIS, NIR1, and NIR2) by plane gratings and a dichroic filter. Its spectral resolution is $6-8 \mathrm{~nm}$, and its $\mathrm{S} / \mathrm{N}$ equal to or better than 2000 around pyroxene absorption band of $1000 \mathrm{~nm}$ (Matsunaga et al., 2000).

This paper presents the performance, objectives and other characteristics of the MI that will observe the mineral distribution on the lunar surface. 


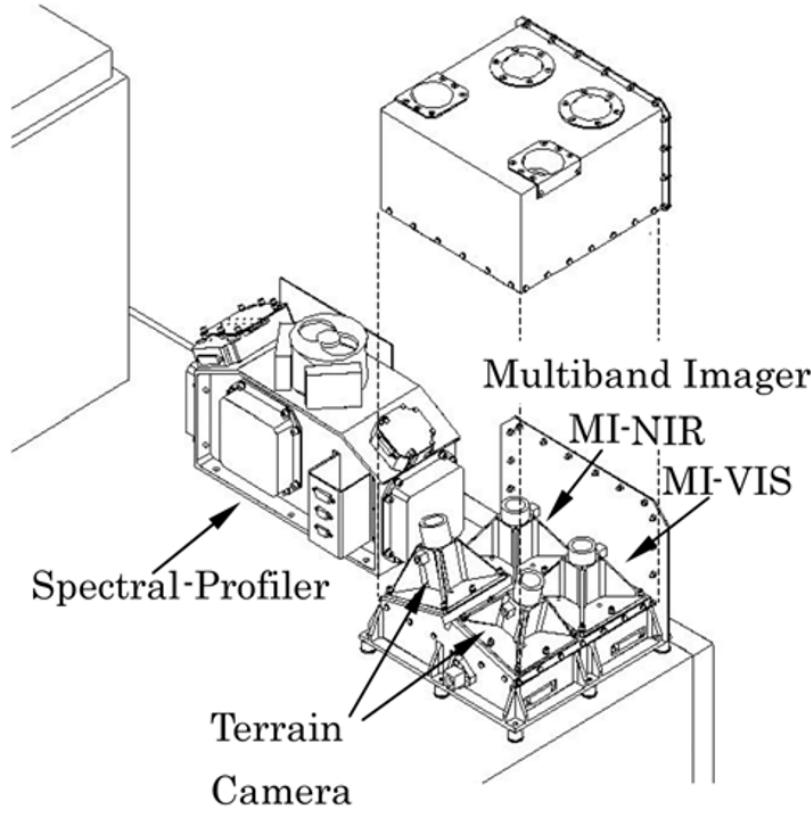

Fig. 1. LISM Radiometer Unit (LRU) of MI/TC and SP on the SELENE (KAGUYA) mission module.

\section{Performance and Design}

MI is a high-resolution multiband imaging camera consisting of visible and near-infrared sensors which have 5 visible and 4 near-infrared bands respectively. Each sensor has the same type of nadir-directed telescope of $65 \mathrm{~mm}$ in focal length but different two-dimension detectors. The spatial resolution of visible bands is $20 \mathrm{~m}$, and that of near-infrared bands is $62 \mathrm{~m}$ from the $100 \mathrm{~km}$ SELENE (KAGUYA) orbital altitude. The swaths of visible and nearinfrared bands are designed to be the same $(19.3 \mathrm{~km}$ from SELENE's $100 \mathrm{~km}$ altitude). MI uses a frame-transfer SiCCD for visible bands and an uncooled InGaAs sensor for near-infrared bands. MI acquires push-broom imaging data by downloading selected lines (962 pixels $\times 5$ lines for visible and 320 pixels $\times 4$ lines for near infrared) of area arrays. The signal resolutions after A/D conversion (and before compression of visible bands) are 10 bits for visible and 12 bits for near-infrared bands. The volume of generated MI images is about $50 \mathrm{Gbit} /$ day (about 6 Gbyte/day) after data compression of visible bands. The spectral band assignments are 415, 750, 900, 950 and $1000 \mathrm{~nm}$ for visible wavelengths and 1000, 1050, 1250 and $1550 \mathrm{~nm}$ for near-infrared wavelengths. The total transmittance of MI, which is calculated as the product of the transmittance of MI optics, transmittance and bandwidth of band-pass filters (BPFs) for each wavelength, and quantum efficiency of used detectors, is controlled to achieve a uniform output signal within each detector. Output uniformity within one detector is important for MI because only one electrical gain can be applied for one detector. The radiation resistance of $\mathrm{MI}$ is achieved using $\mathrm{CeO}_{2}$ doped radiation resistant lenses and also selecting radiation-resistant detectors by performing radiation tests. Table 1 summarizes MI's specifications. Figure 2 depicts MI's observation bands, and Fig. 3, its physical band arrangement. To develop small, simple and light weight multiband imaging systems, we designed a visible detector in which five BPFs are directly attached on the CCD pixel surface and four BPFs are attached on the detector windows for the near-infrared detector. To minimize the smear signal ("smear signal" is a term used for additional exposure during CCD read out), most visible sensor surfaces are shaded by coating except for several window lines for each band (the surface of the near-infrared sensor does not need a shade coating because it is not exposed to light during read out). Additionally, shade coatings, Chromium for visible wavelength and $\mathrm{Ti}+\mathrm{SiO}_{2}$ for near-infrared wavelength are applied to the surfaces of each band-pass filter to avoid stray light and cross-talk between bands except window area for observation. Because MI acquires push-broom imaging data by downloading selected lines of area arrays and use them as if they are multiple line arrays as described above, the observed band images derived from one area array contain parallax between themselves. The area observed by each band at each exposure is different for visible bands because an exposure takes place simultaneously within the visible area array according to the electrical driving algorithm of our CCD. In contrast, the observed area of each near-infrared band is ideally the same if satellite vibration is excluded because the exact timing of each exposure needs to be slightly different for each band according to the electrical driving algorithm of our nearinfrared sensor and we set the difference as the observed area of each band to be same by considering satellite flying speed. In data analyses of MI visible bands, we need to rearrange each band image to the same observed area and perform frame transfer correction (which is caused by exposure under non-shaded window lines). MI does not have an onboard calibration mechanism because of resource limitations. Therefore, cross calibration with SP and multiple observations of well known standard sites are required to achieve reliable data analyses.

\section{Scientific Objectives of the MI}

One of the most important scientific goals of MI is to investigate small but scientifically very important areas such as crater central peaks and crater walls by utilizing MI's high-spatial resolution and high $\mathrm{S} / \mathrm{N}$, combining full spectral coverage data of SP. Tompkins and Pieters (1999) demonstrated the possibility of olivine-rich thin layers (less than $1 \mathrm{~km}$ ) at the crater central peaks of Tsiolkovsky crater. They also demonstrate that, by studying central peaks of major craters of the entire lunar surface, we can extract information about the horizontal and vertical structure of the lunar crustal chemical composition. Therefore, investigations of such small areas will help answer current questions about the existence, chemical composition and source of olivine at the central peaks of some craters and facilitate understanding of the chemical composition within the lunar crust. MI's high spatial resolution will be a most powerful tool for observing the distribution and detailed structures of such thin layers. In addition to its high resolution, MI has the advantage that we can remove topographic effects that cause false reflectance values seen in the crater wall and crater central peak by photometric correction with detailed topography. For example, Robinson and Jolliff 
Table 1. Instrument specification of LISM/MI.

\begin{tabular}{|c|c|c|}
\hline & VIS & NIR \\
\hline Focal length & $65 \mathrm{~mm}$ & $65 \mathrm{~mm}$ \\
\hline F number & 3.7 & 3.7 \\
\hline Field of view & $11 \mathrm{deg}$ & $11 \mathrm{deg}$ \\
\hline Spatial resolution & $20 \mathrm{~m}$ & $62 \mathrm{~m}$ \\
\hline Swath width on ground & $19.3 \mathrm{~km}$ & $19.3 \mathrm{~km}$ \\
\hline Detector & 2D CCD $(1024 \times 1024$ pixel $)$ & $2 \mathrm{D}$ InGaAs $(320 \times 240$ pixel $)$ \\
\hline Pixel size & $13 \times 13 \mu \mathrm{m}$ & $40 \times 40 \mu \mathrm{m}$ \\
\hline Detector cooler & N/A & N/A \\
\hline Number of band & 5 & 4 \\
\hline Band assignment & $415 \pm 10 \mathrm{~nm}$ & $1000 \pm 15 \mathrm{~nm}$ \\
\hline & $750 \pm 5 \mathrm{~nm}$ & $1050 \pm 15 \mathrm{~nm}$ \\
\hline & $900 \pm 10 \mathrm{~nm}$ & $1250 \pm 15 \mathrm{~nm}$ \\
\hline & $950 \pm 15 \mathrm{~nm}$ & $1550 \pm 25 \mathrm{~nm}$ \\
\hline & $1000 \pm 20 \mathrm{~nm}$ & - \\
\hline Quantization & 10 bit & 12 bit \\
\hline $\mathrm{S} / \mathrm{N}$ & $>100$ & $>300$ \\
\hline MTF & >0.2@ Nyquist & >0.2@ Nyquist \\
\hline Integration times & $5.3,2.7$ and $1.3 \mathrm{msec}$ & $26.4,13.2$ and $6.4 \mathrm{msec}$ \\
\hline Data compression & DPCM (loss-less) & N/A \\
\hline Compression rate & $<80 \%$ & - \\
\hline Solar elevation angle in operation & \multicolumn{2}{|c|}{ 30-90 deg } \\
\hline Data amount & \multicolumn{2}{|c|}{49.0 Gbit/day } \\
\hline Mass & \multicolumn{2}{|c|}{$10.2 \mathrm{~kg}$ (as total MI/TC radiometer unit) } \\
\hline Power & \multicolumn{2}{|c|}{$3 \mathrm{~W}$ (as MI radiometer unit and excluding heater) } \\
\hline
\end{tabular}

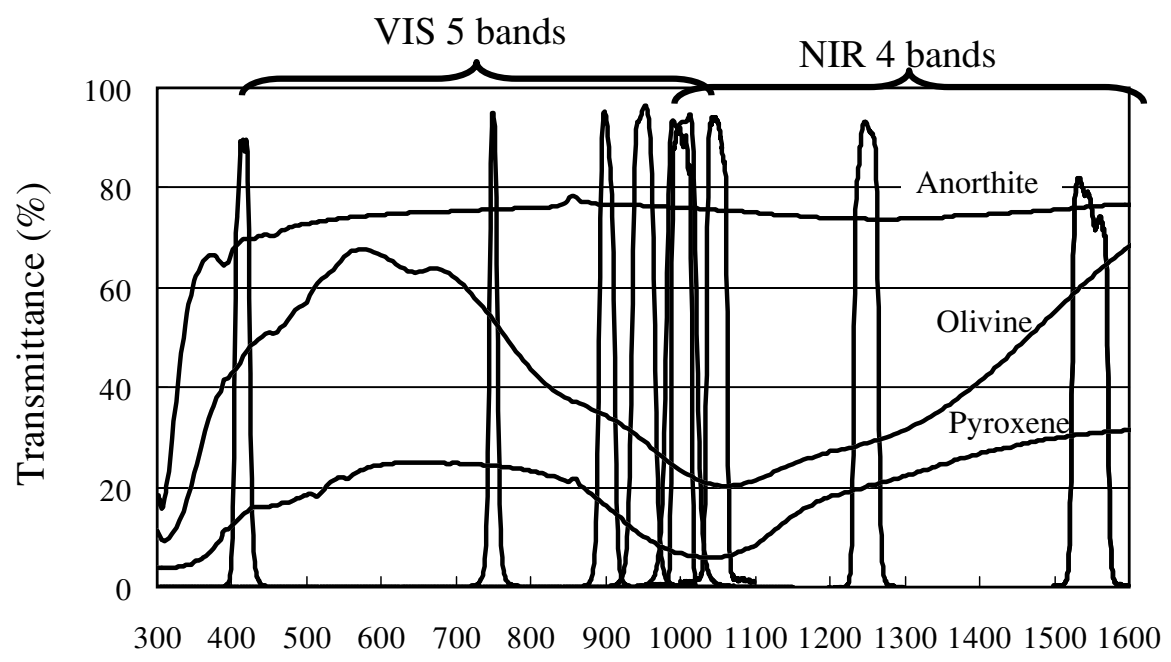

Wavelength (nm)

Fig. 2. MI observation bands with examples of mineral reflectance (terrestrial samples) of major lunar surface mineral species. MI's observation bands are shown as a transmittance curve for each band-pass filter.

(2002) demonstrated that $\mathrm{FeO}$ and $\mathrm{TiO}_{2}$ abundances calculated using methods described by Blewett et al. (1997), Lucey et al. (1998), Jolliff (1999), and Lucey et al. (2000a, b) can change as much as $5 \mathrm{wt} \%$ (FeO; absolute) and $4 \mathrm{wt} \%$ $\left(\mathrm{TiO}_{2}\right.$; absolute) on 30-degree slopes even though the average slope of the crater central peak may not be so steep. Digital terrain models (DTMs) for topographic correction are derived from TC stereoscopic images or MI band sets that have 11.2 degree maximum parallax (parallax between nearest band sets are about 3-4 degree).

MI's low stray light (both spatial and spectral) is also very useful for investigating dark areas within bright regions such as dark mantling material and highland mare, although it may be better to investigate these areas with optimized exposure settings. One more important objective of $\mathrm{MI}$ is to search for previously unidentified lunar crustal rock similar to the magnesian anorthosites recently found in lunar meteorite Dho489 by Takeda et al. (2003, 2006). They found that Dho489 contains clasts in which there are more Mg-rich mafic minerals (both olivine and pyroxene) than Ferroan Anorthosites (FAN), which is widely considered as a typical highland material, and the clasts are not mixture of 


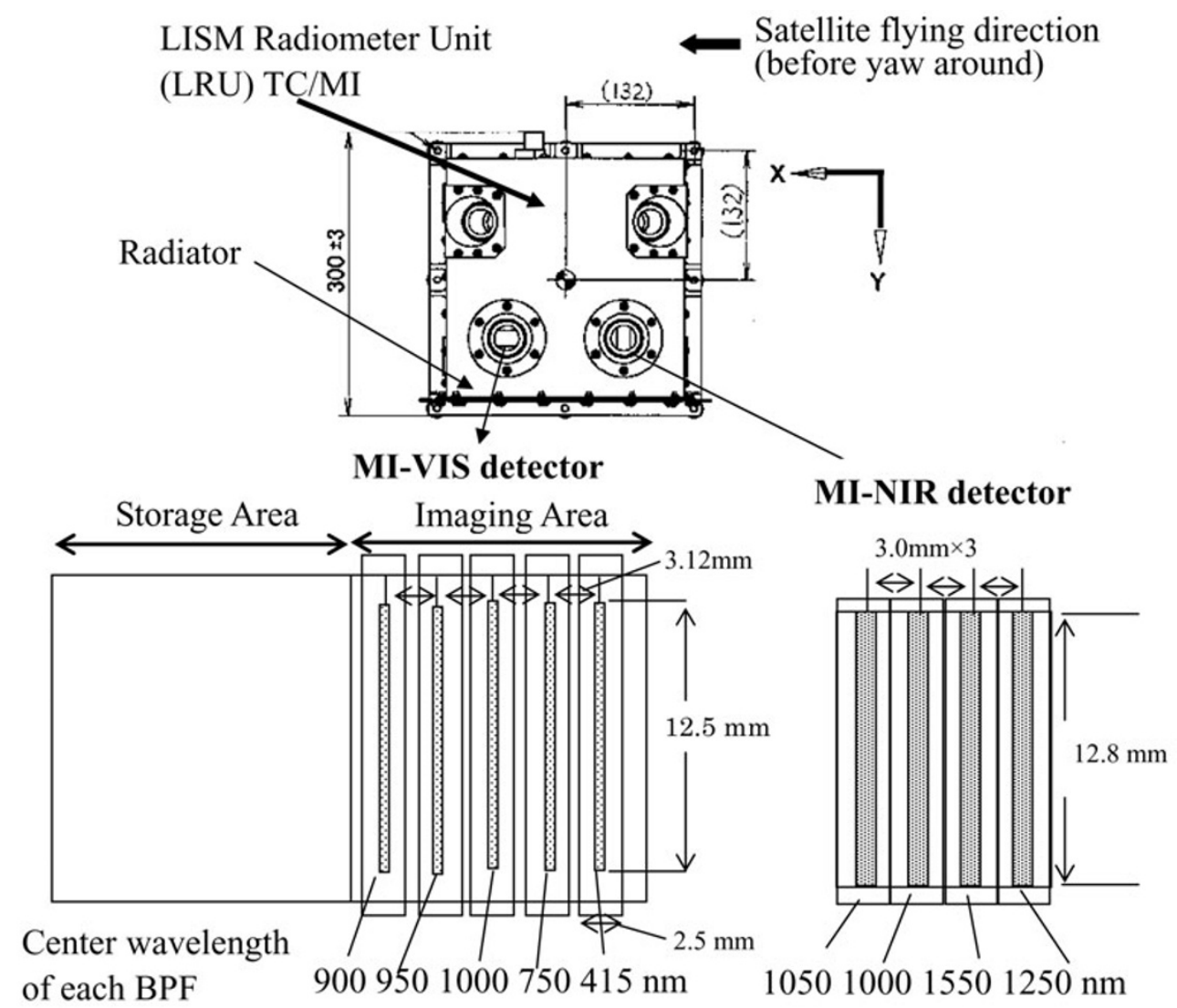

Fig. 3. Physical band arrangement of MI. Arrangement is shown as viewed from the Moon direction.

two components (Mg-suite and FAN). It is also suggested that Dho489 comes from the lunar far side. This finding suggests the possible existence of magnesian anorthosites on the lunar far side. If this is true, the understanding of its distribution and chemical composition is very important for acquiring new constraints for the lunar crustal growth model.

\section{Manufacturing and Pre-flight Test}

The MI flight model has been manufactured and integrated (Fig. 4), and preflight testing of the SELENE (KAGUYA) satellite is underway. The MTF, viewing vector, over-all sensibility, sensor linearity and electrical noise level (S/N estimation) were measured after the MI integration. Results of the MI preflight testing will be discussed in more detail in a paper by Kodama and Ohtake (in preparation), but measured data indicate that MI will provide sufficient MTF and low-noise data, just as estimated in the MI design phase. Furthermore, as a result of continuous effort, cross talk among spectral bands has been kept especially low.

\section{Operation Plan}

MI take nine-band images of the entire surface of the Moon during the one-year SELENE (KAGUYA) nominal mission. Visible images are compressed using a lossless algorithm by an onboard compression chip, while nearinfrared images are not compressed. Figure 5 presents an example of an annual MI operation plan during a nominal SELENE (KAGUYA) mission. The annual operation plan

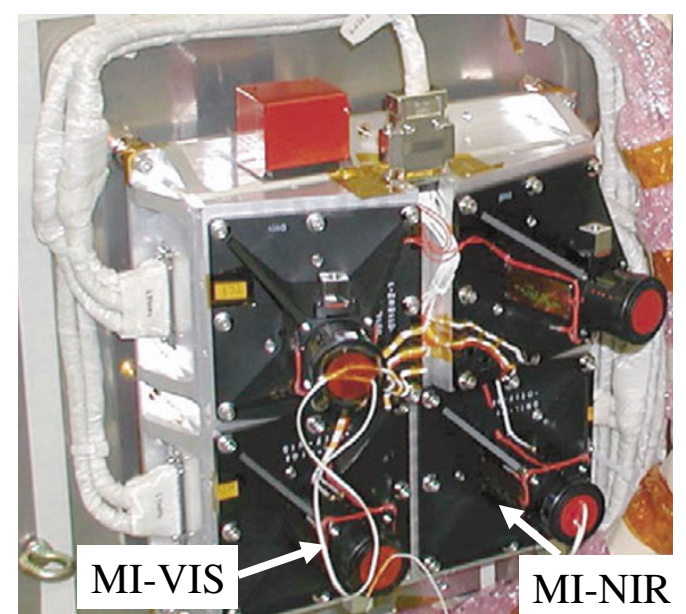

Fig. 4. Flight model of LISM Radiometer Unit (LRU) of MI/TC attached to the dummy satellite panel. MI and TC sensors share a unit structure. An optical hood is attached above the sensors in the actual flight configuration.

changes according to the $\beta$ angle of the starting point of the nominal observation phase. During its nominal mission period, MI image 1/3 of the Sun-illuminated area of each orbit in the standard observation mode (observation using the nominal lossless compression algorithm) and image the remaining 2/3 of each orbit in the SP support mode (observation using lossy and high-compression algorithm) to control the data volume to be down linked and also to provide 


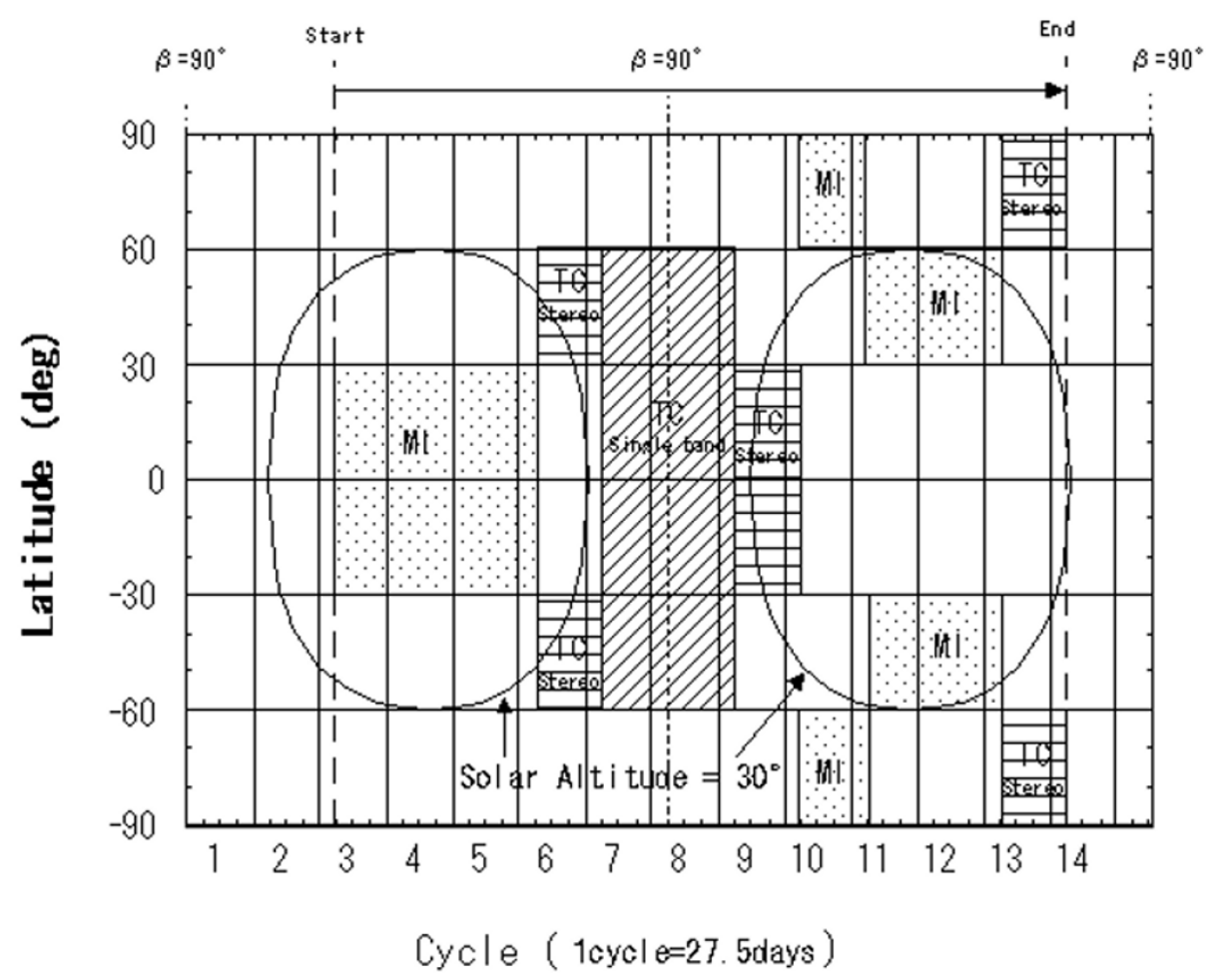

Fig. 5. Example of annual MI operation plan during SELENE (KAGUYA) nominal mission. The annual operation plan changes according to the $\beta$ angle of the start point of the nominal observation phase. MI will make observations at solar elevation angles from $30^{\circ}$ to $90^{\circ}$. MI uses six cycles to cover the entire Moon because its swath is narrower than each orbit gap and the down-linkable data volume is limitted.

images for SP to determine its observation profile. For this reason, MI will observe low (0 to 30 degrees), middle (30 to 60 degrees) and high (60 to 90 degrees) latitudes in different observation sequences. MI's swath width $(19.9 \mathrm{~km}$ from the nominal $100 \mathrm{~km}$ SELENE orbital altitude) is narrower than the nominal interval of SELENE's orbit, and therefore SELENE's orbit plane will be changed three times (twice for low latitudes and once for middle latitudes) to cover the entire lunar surface while maintaining overlap between each strip by MI. To cover the entire surface of the Moon, MI needs six observation sequences in total. TC and MI will make complementary observations to optimize the solar elevation angle (30 to 90 for MI) for each instrument and to minimize electric power consumption. During nominal observation sequences, an exposure time will be selected according to the surface reflectance and phase angle from three exposure modes (short, middle and long; their exposure time ratio is $4: 2: 1$ ) to achieve sufficient signal count and also to prevent signal saturation.

In addition to the nominal observation plan designed to achieve unsaturated images of the entire lunar surface, we are going to take some shaded area images for on-board dark-current correction throughout the mission period to obtain data under a wide range of temperatures. Also, additional data can be obtained depending on the excess data transmission capacity. The possibility of obtaining detailed images of permanently shadowed and illuminated areas in the polar region and obtaining basic data to investigate opposition effects are being investigated.

We are also planning to take several images of our standard site, which is located near the Apollo 16 landing site and has been used as the standard site for many previous Earth-based telescopic observations as well as the Clementine UVVIS camera (Nozette et al., 1994), throughout the mission period to check (and if necessary, calibrate) sensor sensitivity. Such multiple observations of the standard site are very important for MI data analyses because MI has only limitted opportunities to observe the same area under different phase angle conditions. Studies to select the best optical standard site for MI and to derive accurate reflectances of selected standard sites using the standard site data are in progress as described in Ohtake et al. (2005).

\section{Data Analyses Plan}

On-ground LISM data processing systems have been established as a subsystem of the SELENE Operation and Analyses Center (SOAC) on JAXA's Sagamihara Campus. Most of the interface tests between other systems and endto-end tests (from generation of an instrument operation plan to LISM data analyses) have been finished and have confirmed the system efficiency. The data storage and distribution system for all SELENE (KAGUYA) mission data is also installed in SOAC.

Figure 6 depicts MI's ground data processing flow. Each step of the data processing flow will be discussed below. LISM data will be down-linked from the SELENE (KAGUYA) satellite to the Earth in real time or stored in an onboard mission data recording system. For our data analyses, we use SPICE kernels and the NAIF toolkit developed in JPL.

We are going to produce Level 2A, Level 2B, Level 2C and MAP products using our data processing systems. 


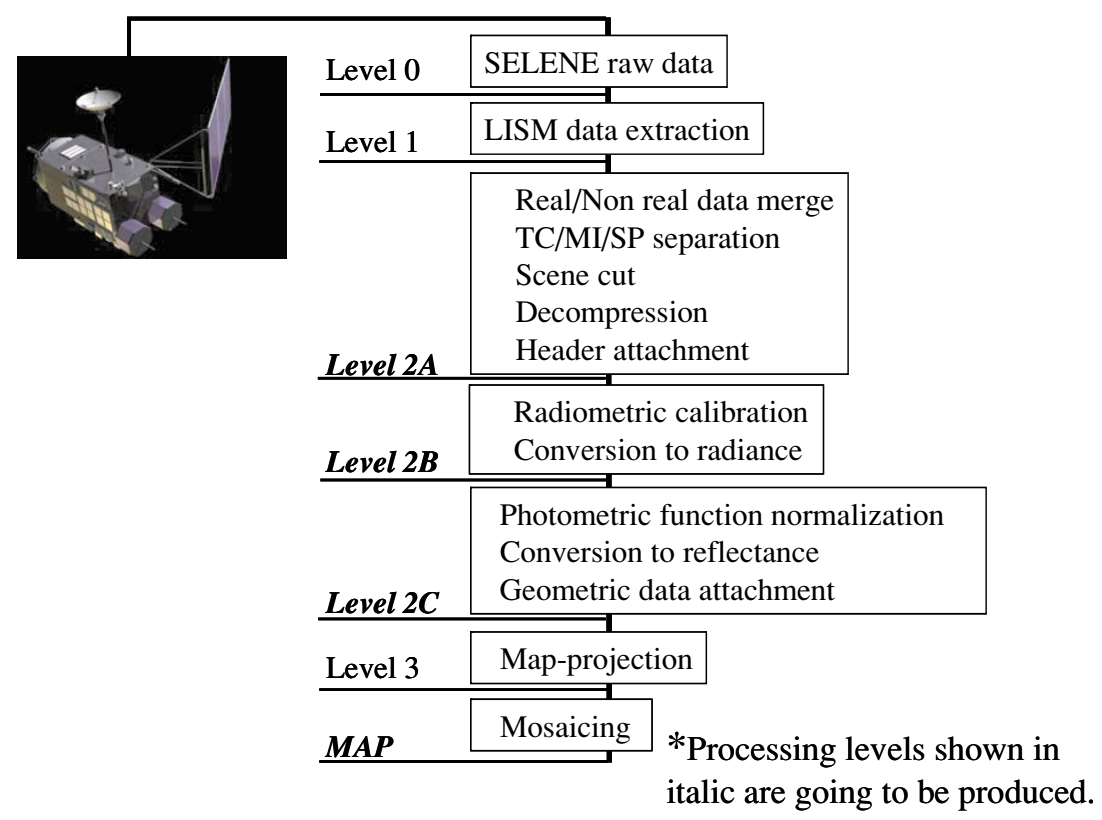

Fig. 6. Overview of MI's ground data processing flow.

These products will be distributed first to the LISM and SELENE (KAGUYA) team and then to the public according to the SELENE (KAGUYA) data distribution plan. Distribution of SELENE (KAGUYA) data will start one year after nominal one-year mission. Level 2A and Level 2B products that cover the entire Moon will be produced, and Level 2C and higher processed products will first be produced to satisfy the needs of each science topic proposed by LISM science members. Table 2 lists products created from MI data. All LISM products will be distributed in a PDS-like format.

a-1) Radiometric correction: Radiometric correction of MI will be carried out according to the equation shown below.

$$
\mathrm{RCOR}=\frac{f_{\mathrm{NLT}}(\mathrm{SVAL}-\mathrm{FT})}{\mathrm{FLAT} * \mathrm{EFFIC}}
$$

SVAL: SVAL $=$ RAW - DARK (RAW is a raw signal of MI, and DARK is a dark-current correction coefficient generated for each pixel of each band. DARK will be derived using observation data of shaded areas.)

RCOR: Value after radiometric correction.

$f_{\mathrm{NLT}}$ : Function for on-linearity correction of detector. $f_{\mathrm{NLT}}$ is determined from MI data under different (known) input radiance using an integrated sphere which was taken during the pre-flight optical testing.

FLAT: Correction coefficient for flat fields. FLAT will be created using on board data by adding and normalizing significant amounts of images taken randomly.

EFFIC: Correction coefficient of transmittance temperature dependency. EFFIC is determined from MI data under different temperature condition which was taken during the pre-flight optical testing.

FT: Correction values of frame transfer (only visible bands need this correction). FT will be created using on board imaging data by calculating additional signal during frame transfer under non-shaded window lines of each exposure from calculated ratio of intended exposure time and additional exposure time during frame transfer. a-2) Conversion to radiance: After the radiometric correction radiance of each pixel data is calculated after normalization in time according to the exposure setting of each image using the conversion coefficient of each band from our pre-flight optical testing. Conversion coefficient of each band is determined from MI data observing a known radiance target (integrated sphere) which was taken during the pre-flight optical testing. Calculated radiance data with its attached header is called Level 2B.

b-1) Photometric function normalization and conversion to reflectance: After solar distance normalization, the data is normalized to a standard geometry $(i=30$ and $e=0$ ) using a photometric function adapted according to the observation geometry, which is calculated for each image pixel. We are going to adopt the photometric function and its parameters for each band used in McEwen (1996) and McEwen et al. (1998) during our initial data processing period to compare MI data to Clementine UV/VIS camera images. Photometric function parameters for longer than $1000 \mathrm{~nm}(1050,1250$ and $1550 \mathrm{~nm})$ are not available in McEwen (1996) and McEwen et al. (1998). Therefore we are going to determine the parameters in our ongoing study. MI can adapt topographic correction to do photometric function normalization based on the three different geometric calibration options calculated in step b-3.

b-2) Conversion to reflectance: Reflectance of each pixel data is calculated using conversion coefficients derived by either our pre-flight optical test or by comparison of the reflectance of standard sample(s) measured in a laboratory and values observed at the standard site. We use MODTRAN.thkur (Thuillier et al., 1997, 1998) as the incoming solar flux to calculate conversion coefficients derived by pre-flight optical testing. We are preparing laboratory measured reflectance for standard samples as discussed in Ohtake et al. (2005).

b-3) Geometric data attachment: Our geometric correction algorithm has three options, and we can select one of 
Table 2. MI product list. Each product listed below is going to be produced and stored in L2DB in SOAC.

\begin{tabular}{|c|c|c|c|c|}
\hline $\begin{array}{l}\text { Process } \\
\text { level }\end{array}$ & Product name & Resolution & Unit & Product detail \\
\hline \multirow{4}{*}{ L2A } & MI-VIS_Level2A & $20 \mathrm{~m} / \mathrm{pxl}$ & $\operatorname{raw}[\mathrm{DN}]$ & $\begin{array}{l}\text { MI-VIS } 5 \text { band images in nominal observation mode. After depacket, } \\
\text { sort by time and scene cutting. No correction and calibration were } \\
\text { applied. Compressed as } 12 \text { bit JPG file. }\end{array}$ \\
\hline & MI-NIR_Level2A & $60 \mathrm{~m} / \mathrm{pxl}$ & $\operatorname{raw}[\mathrm{DN}]$ & $\begin{array}{l}\text { MI-NIR } 4 \text { band images in nominal observation mode. After depacket, } \\
\text { sort by time and scene cutting. No correction and calibration were } \\
\text { applied. Uncompressed data. }\end{array}$ \\
\hline & MI-VIS_SPsupport_Level2A & $160 \mathrm{~m} / \mathrm{pxl}$ & $\operatorname{raw}[\mathrm{DN}]$ & $\begin{array}{l}\text { MI-VIS } 1 \text { band images in SP support observation mode. After depacket, } \\
\text { sort by time and scene cutting. No correction and calibration were } \\
\text { applied. Compressed as } 12 \text { bit JPG file. }\end{array}$ \\
\hline & MI-NIR_SPsupport_Level2A & $480 \mathrm{~m} / \mathrm{pxl}$ & raw $[\mathrm{DN}]$ & $\begin{array}{l}\text { MI-NIR } 1 \text { band images in SP support observation mode. After depacket, } \\
\text { sort by time and scene cutting. No correction and calibration were } \\
\text { applied. Compressed as } 12 \text { bit JPG file. }\end{array}$ \\
\hline L2B & MI-VIS_Level2B2 & $20 \mathrm{~m} / \mathrm{pxl}$ & $\begin{array}{l}\text { radiance } \\
{\left[\mathrm{w} / \mathrm{m}^{2} / \mu \mathrm{m} / \mathrm{sr}\right]}\end{array}$ & $\begin{array}{l}\text { MI-VIS } 5 \text { band images in nominal observation mode. After radiometric } \\
\text { correction, conversion to radiance, rubber seating of non-base images } \\
\text { to the base images, scene cutting as same observation area and cube } \\
\text { generation. }\end{array}$ \\
\hline L2B & MI-NIR_Level2B2 & $60 \mathrm{~m} / \mathrm{pxl}$ & $\begin{array}{l}\text { radiance } \\
{\left[\mathrm{w} / \mathrm{m}^{2} / \mu \mathrm{m} / \mathrm{sr}\right]}\end{array}$ & $\begin{array}{l}\text { MI-NIR } 4 \text { band images in nominal observation mode. After radiometric } \\
\text { correction, conversion to radiance, rubber seating of non-base images } \\
\text { to the base images, scene cutting as same observation area and cube } \\
\text { generation. Data values are shown in radiance. }\end{array}$ \\
\hline $\mathrm{L} 2 \mathrm{C}$ & MI-VIS_Level2C2 & $20 \mathrm{~m} / \mathrm{pxl}$ & $\begin{array}{l}\text { reflectance } \\
\text { [non-dimension] }\end{array}$ & $\begin{array}{l}\text { MI-VIS } 5 \text { band images in nominal observation mode. After photometric } \\
\text { correction, conversion to reflectance and attachment of systematic } \\
\text { geometric correction data (latitude and longitude derived by geometric } \\
\text { correction). }\end{array}$ \\
\hline $\mathrm{L} 2 \mathrm{C}$ & MI-NIR_Level2C2 & $60 \mathrm{~m} / \mathrm{pxl}$ & $\begin{array}{l}\text { reflectance } \\
\text { [non-dimension] }\end{array}$ & $\begin{array}{l}\text { MI-NIR } 4 \text { band images in nominal observation mode. After photometric } \\
\text { correction, conversion to reflectance and attachment of systematic } \\
\text { geometric correction data (latitude and longitude derived by geometric } \\
\text { correction). }\end{array}$ \\
\hline MAP & MI_MAP & $\begin{array}{l}2^{11} \mathrm{pixel} / \text { degree } \\
2^{7} \mathrm{pixel} / \text { degree }\end{array}$ & $\begin{array}{l}\text { reflectance } \\
\text { [non-dimension] }\end{array}$ & $\begin{array}{l}\text { Mosaic data after } 9 \text { band cube generation and map projection (simple } \\
\text { cylindrical). For mosaicing image matching are applied to overlapping } \\
\text { area of the original images. }\end{array}$ \\
\hline
\end{tabular}

them to suit the requirements for each scientific purpose and time available for data analyses. To normalize the photometric function and perform geometric calibration and map-projection, we need the following geometric parameters for each pixel: 1) Latitude and either altitude or threedimensional position of each pixel in the lunar centric coordinate system, 2) Illumination angle, emission angle and phase angle of each pixel, 3) Moon-Sun distance. To determine these parameters four different options of geometric calibrations are possible. Options of geometric calibration are as follows.

Option 1

- Calculate geometric parameters by estimating a spherical lunar surface, and apply systematic geometric calibration (calculated from the measured orbit and altitude of the satellite, viewing vector of each pixel, and time of each exposure) for all images. In this option registration within band images does not achieve and local topographic features are neglected and therefore reflectance is calculated without topographic correction.

\section{Option 2}

- Calculate geometric parameters by estimating a spherical lunar surface, perform rubber seating (rubber seating means deforming several band images to match one selected base image), and then apply systematic geometric calibration for the base image. This option achieves registration within band images, but local topographic features are neglected and therefore reflectance is calculated without topographic correction.

\section{Option 3}

- Calculate geometric parameters by using both TC ortho image mosaics and DTMs created from TC stereo images. In this option, reflectance is calculated with topographic correction.

\section{Option 4}

- Calculate geometric parameters by image matching within MI cubed bands and using topographic data from MI images. In this option, reflectance is calculated with topographic correction.

Calculated reflectance data with attached geometric data 
(latitude and longitude of each pixel) and header is called as Level $2 \mathrm{C} 2 / 3 / 4$. The number attached after " $\mathrm{C}$ " indicates the option number used for geometric parameter calculation. We are going to use option 2 in the first stage of MI data analyses and provide it to the public because it can be applied uniformly for the entire data set of MI observations and it shortens the time required for data analyses (option 1 is applied only for TC).

\section{Preflight Evaluation of Geometric Calibration}

We evaluated the accuracy of our geometric and topographic calibration by comparing modeled topographic features (used as "real values" of the lunar surface generated by adding small crater features to base DEM data, to derive geometric data (latitude and longitude of each pixel) and topographic features (calculated "slope" at each pixel) from simulated MI band images of different geologic settings (mare, highland and central peak), solar altitude, and solar angle conditions. In this evaluation, the effects of various errors are considered such as hardware characteristics ( $\mathrm{S} / \mathrm{N}$, flat-field and viewing vector with their measurement errors), number of added small craters, errors of satellite positioning, attitude determination, and errors caused by highfrequency vibration. Our evaluation results indicate that the accuracy of geometric calibration (except for local topographic features) using any option is sufficiently high and errors of the slope after geometric correction is less than 5 degree for options 3 and 4 except for extremely low solar altitudes. Our evaluation may not completely simulate all possible observation conditions and real reflectance variations of the lunar surface, but it clearly demonstrates the efficiency of the topographic correction.

\section{Summary}

MI is designed to be a high-resolution multiband imaging camera with a spatial resolution in visible bands of $20 \mathrm{~m}$, and the resolution of near-infrared bands is $62 \mathrm{~m}$ from the $100 \mathrm{~km}$ SELENE (KAGUYA) orbital altitude. Optical tests were conducted, and the results indicate that MI will provide sufficient MTF and low-noise data. Tools and algorithms for operation and data analyses have been developed and checked. An example of MI scientific goals is to investigate small but scientifically very important areas such as crater central peaks and crater walls and to investigate magnesian anorthosites.

Acknowledgments. We thank SELENE (KAGUYA) mission team members including all instrument science team members for useful discussions beneficial to our instrument design, operation planning, and planning of data analyses. We also thank FJT Limited engineers T. Maekawa, K. Tsubosaka and N. Tonoya for hardware development and J. Inoue, N. Masuda and T. Nakashima for Level 2A software development. We are grateful to Mitsubishi Space Software Co., LTD engineers M. Hashimoto, K. Torii, Y. Kurashina and A. Yoshizawa for developing advanced LISM data analyses software. We appreciate the help of Dr. C. Acton for kindly providing us detailed directions for SPICE kernel and NAIF toolkit.

\section{References}

Blewett, D. T., P. G. Lucey, B. R. Hawke, and B. L. Jolliff, Clementine images of the lunar sample-return stations: Refinement of $\mathrm{FeO}$ and $\mathrm{TiO}_{2}$ mapping techniques, J. Geophys. Res., 102, 16319-16325, 1997.

Haruyama, J., T. Matsunaga, M. Ohtake, T. Morota, C. Honda, Y. Yokota, M. Torii, Y. Ogawa, and the LISM Working Group, Global lunar-surface mapping experiment using the Lunar Imager/Spectrometer on SELENE, Earth Planets Space, 60, this issue, 243-255, 2008.

Jolliff, B. L., Clementine UVVIS multispectral data and the Apollo 17 landing site: What can we tell and how well?, J. Geophys. Res., 104, 14123-14148, 1999.

Kato, M., S. Sasaki, K. Tanaka, Y. Iijima, and Y. Takizawa, The Japanese lunar mission SELENE: Science goals and present status, Advances in Space Research, doi:10.1016, (in print).

Kodama, S. and M. Ohtake, (in prep).

Lucey, P. G., D. T. Blewett, and B. R. Hawke, Mapping the $\mathrm{FeO}$ and $\mathrm{TiO}_{2}$ content of the lunar surface with multispectral imagery, J. Geophys. Res., 103, 3679-3699, 1998.

Lucey, P. G., D. T. Blewett, and B. L. Jolliff, Lunar iron and titanium abundance algorithms based on final processing of Clementine ultravioletvisible images, J. Geophys. Res., 105, 20297-20305, 2000a.

Lucey, P. G., D. T. Blewett, G. J. Taylor, and B. R. Hawke, Imaging of lunar surface maturity, J. Geophys. Res., 105, 20377-20386, 2000b.

Matsunaga, T., M. Ohtake, Y. Hirahara, and J. Haruyama, Development of a visible and near infrared spectrometer for Selenological and Engineering Explorer (SELENE), Proceedings of SPIE, 4151, 32-39, 2000.

McEwen, A. S., A precise lunar photometric function, Lunar and Planetary Science, XXVII, \#841, Lunar and Planetary Institute, Houston (CD-ROM), 1996.

McEwen, A. S., E. M. Eliason, P. G. Lucey, E. Malaret, C. M. Pieters, M. S. Robinson, and T. Sucharski, Radiometric calibration and photometric normalization for Clementine, Lunar and Planetary Science, XXIX \#1466, Lunar and Planetary Institute, Houston (CD-ROM), 1998.

Nozette, S., P. Rustan, L. P. Pleasance, D. M. Horan, P. Regeon, E. M. Shoemaker, P. D. Spudis, C. H. Acton, D. N. Baker, J. E. Blamont, B. J. Buratti, M. P. Corson, M. E. Davies, T. C. Duxbury, E. M. Eliason, B. M. Jakosky, J. F. Kordas, I. T. Lewis, C. L. Lichtenberg, P. G. Lucey, E. Malaret, M. A. Massie, J. H. Resnick, C. J. Rollins, H. S. Park, A. S. McEwen, R. E. Priest, C. M. Pieters, R. A. Reisse, M. S. Robinson, R. A. Simpson, D. E. Smith, T. C. Sorenson, R. W. Vorder Breugge, and M. T. Zuber, The Clementine mission to the Moon: Scientific overview, Science, 266, 1835-1839, 1994.

Ohtake, M., T. Arai, and H. Takeda, Study of the Apollo16 landing site: Re-visit as a standard site for the SELENE Multiband Imager, Lunar and Planetary Science, XXXVI, \#1637, Lunar and Planetary Institute, Houston (CD-ROM), 2005.

Robinson, M. S. and B. L. Jolliff, Apollo 17 landing site: Topography, photometric corrections, and heterogeneity of the surrounding highland massifs, J. Geophys. Res., 107, 5110, doi:10.1029/2001JE001614, 2002.

Takeda, H., K. Saiki, T. Ishii, and M. Otsuki, Mineralogy of the Dhofar 489 lunar meteorite, crystalline matrix breccia with magnesian anorthositic clasts, Lunar and Planetary Science, XXXIV, \#1284, Lunar and Planetary Institute, Houston (CD-ROM), 2003.

Takeda, H., A. Yamaguchi, D. D. Bogard, Y. Karouji, M. Ebihara, M. Ohtake, K. Saiki, and T. Arai, Magnesian anorthosites and a deep crustal rock from the farside crust of the moon, Earth Planet. Sci. Lett., 247, 171-184, 2006.

Thuillier, G., M. Hersé, P. C. Simon, D. Labs, H. Mandel, and D. Gillotay, Observation of the UV solar spectral irradiance between 200 and 350 $\mathrm{nm}$ during the ATLAS I mission by the SOLSPEC spectrometer, Solar Physics, 171(2), 283-302, 1997.

Thuillier, G., M. Hersé, P. C. Simon, D. Labs, H. Mandel, D. Gillotay, and T. Foujols, The visible solar spectral irradiance from 350 to $850 \mathrm{~nm}$ as measured by the SOLSPEC spectrometer during the ATLAS I mission, Solar Physics, 177(1-2), 41-61, 1998.

Tompkins, S. and C. M. Pieters, Mineralogy of the lunar crust: Results from Clementine, Meteoritics Planet. Sci., 34, 25-41, 1999.

M. Ohtake (e-mail: ohtake.makiko@jaxa.jp), J. Haruyama, T. Matsunaga, Y. Yokota, T. Morota, C. Honda, and LISM team 